\title{
Rio de Janeiro against Aedes aegypti: yellow fever in 1908 and dengue in 2008 - Editorial
}

In 2008, Brazil is experiencing its second most devastating dengue epidemic, with 734,000 reported dengue cases through October this year. However, this represents the most severe dengue epidemic in terms of deaths: 212 deaths were recorded, primarily in children. Deaths have been mainly reported for infections with dengue virus type 2 (DENV-2) in endemic areas that had previously experienced epidemics of DENV-3. Southeastern and Northeastern Brazilian cities have been most affected, and in some cities, the incidence has increased more than 2,000-fold. Again, the "eye of the hurricane" has been in Rio de Janeiro, located in the Southeast, the most "developed" region of Brazil. A favourable climate for reproduction of the mosquito vector Aedes aegypti, degradation of environmental conditions, low income levels, violence, and drug fiefdoms on hillside slums, political quarrels, have all hampered efficient control activities, leading to a deterioration of public services and minimized integrated activity with regard to sanitation, education, communication, and dengue vector control, resulting in the emergence of Rio de Janeiro as a major point of dengue introduction and dissemination into the country. Additionally, and unfortunately, almost no results from scientific investigations on the dengue vector have been taken into account in local dengue control campaigns.

This is similar to circumstances over 100 years ago in which the physician Antonio Gonçalves Peryassu published a monograph in Rio de Janeiro, whose data encouraged the fight against Ae. aegypti. The two main differences between this historical scenario and the current one are the vector carried virus (yellow fever in 1908, and dengue in 2008) and the strong influence of Oswaldo Cruz, the director of the Instituto de Manguinhos and yellow fever mosquito campaigns that resulted in implementation of laboratory and field scientifically proven results in the mosquito control strategies.

Peryassu's monograph entitled "Os Culicideos do Brasil" (Culicids from Brazil) is comprised of 407 pages containing more than 60 black and white and colour plates, as well as a detailed mosquito map of almost every street in the city of Rio de Janeiro in 1906. This comprehensive work on Brazilian and South American mosquito systematics and biology was produced over a period of two years of investigation at the Instituto de Manguinhos, which was named Instituto Oswaldo Cruz in 1908. Peryassu's work was clearly driven by the need for scientific knowledge regarding Ae. aegypti biology and behaviour. Transmission of the yellow fever virus by Ae. aegypti was discovered only few years earlier Peryassu's first field and laboratory assays, which were initiated in 1906. Prior to this work, all that was known about Ae. aegypti's behaviour and biology had been reported by the US Army Yellow Fever Commission in Cuba. Almost nothing was known about Ae. aegypti behaviour and biology in the environmental and climatic conditions of Rio de Janeiro. In addition to the substantial data describing Stegomyia calopus (=Ae. aegypti) and other mosquito species provided in three large chapters in the first section of his monograph, Peryassu also described his numerous laboratory experiments examining the biology of this mosquito in the second part of the book. He sought to discover the weakness in the mosquito life cycle that could be exploited in control campaigns. Some of the main assays and field observations described in Peryassu's work include resistance of eggs, larvae, and pupae to desiccation, co-habitation of Ae. aegypti with other immature native mosquitoes in several breeding environments, and larval and pupal mortality due to exposure to seawater, sodium chloride, and mercury chloride. He also pointed out that the most productive habitats for immature Ae. aegypti were large and permanent water-holding containers. Peryassu demonstrated that Ae. aegypti larvae survived exposure to soap at concentrations regularly used in tanks for washing clothes (and subsequently held in drains). Furthermore, he found that when the local temperature dropped below $20^{\circ} \mathrm{C}$, Ae. aegypti development and reproduction were strongly affected. "In nature, a single night with temperature dropping to $20^{\circ} \mathrm{C}$ is enough to delay and disrupt larval hatching and development", reinforced Peryassu. Additionally, Peryassu's incredibly detailed map of the distribution Ae. aegypti in the city of Rio de Janeiro (supplementary data) shows that the mosquito distribution is clearly influenced by human population density. Indeed, Peryassu emphasized that Ae. aegypti was considerably more frequent in the populated city centre and that its distribution matched that of yellow fever cases.

Peryassu's monograph was assembled under the guidance of Arthur Neiva, with some advices from Adolpho Lutz. He received encouragement and sponsorship from Oswaldo Cruz, who was particularly interested in mosquito studies and the role of mosquito species in human disease transmission. Oswaldo Cruz himself wrote (and possibly illustrated) the first paper published at the Instituto Oswaldo Cruz on a new species of anopheline. In 1903, Oswaldo Cruz also launched the anti-yellow fever mosquito campaign, which culminated in the control of yellow fever epidemics in Rio late in the 1920s. Ae. aegypti was considered eradicated from Brazil in 1955 after a continental campaign.

Currently, 100 years later, Rio's main enemy is once again Ae. aegypti. Again, the battle must be won not only by the health authorities and governmental anti-mosquito campaigns, but also by scientists. Since a dengue vaccine is still not available, control strategies have been focused on mosquito population reduction. An efficient campaign against Ae. aegypti must be based on scientific investigations, taking into account that large metropolitan areas such as Rio have spatial heterogeneity in terms of the vector population. This obviously makes control strategies more complex. Additionally, dengue control will require much more than a health-based approach. For instance, the 
strategy must be constructed with inputs and actions from education and sanitation backgrounds. Scientific investigations, especially those conducted in networks with multidisciplinary approaches, must be utilised as the primary method of gaining information in support of an efficient control campaign in Rio de Janeiro. As Oswaldo Cruz and AG Peryassu suggested 100 years ago, funding of scientific investigations and promotion of interactions between the authorities involved in control at all levels and scientific workers is essential in the fight against mosquitoes during the next dengue transmission season.

Ricardo Lourenço-de-Oliveira 


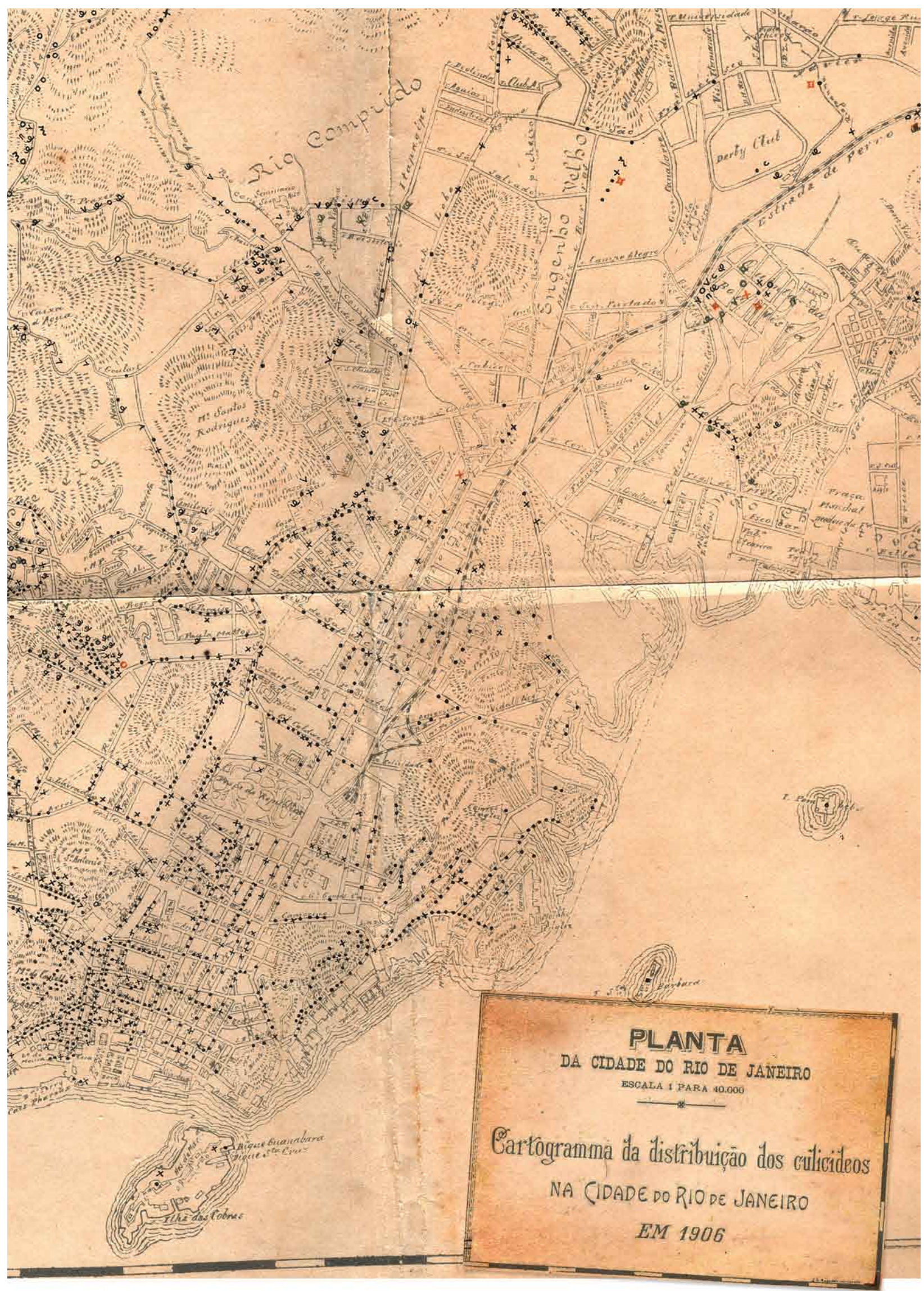

Section of Peryassu's map of Aedes aegypti and other mosquito species distribution in the city of Rio de Janeiro, in 1909. 